EPJ Web of Conferences 37, 09036 (2012)

DOI: $10.1051 /$ epjconf/20123709036

C) Owned by the authors, published by EDP Sciences, 2012

\title{
Parameters of scalar resonances from the combined analysis of $\pi \pi$ scattering and coupled-channel data
}

\author{
Yu.S. Surovtsev ${ }^{1, a}$, P. Bydžovský ${ }^{2}$, T. Gutsche ${ }^{3}$, V.E. Lyubovitskij ${ }^{3}$, R. Kamiński ${ }^{4}$, and M. Nagy ${ }^{5}$ \\ 1 Bogoliubov Lab of Theoretical Physics, JINR, Dubna, Russia \\ 2 Nuclear Physics Institute, ASCR, Rež/Prague, Czech Republic \\ 3 Institut für Theoretische Physik, Universität Tübingen, Germany \\ ${ }^{4}$ Institute of Nuclear Physics, PAN, Krakow, Poland \\ 5 Institute of Physics, SAS, Bratislava, Slovak Republic
}

\begin{abstract}
Peculiarities of obtaining parameters for broad multichannel resonances from data are discussed analyzing the experimental data on processes $\pi \pi \rightarrow \pi \pi, K \bar{K}$ in the scalar channel in a model-independent approach based on analyticity and unitarity and using an uniformization procedure. It is shown that one can obtain a good description of the $\pi \pi$ scattering data from the threshold up to $1.89 \mathrm{GeV}$ with values of resonance parameters cited in the PDG tables as preferred. However, in this case the representation of the $\pi \pi$ background is not satisfactory and the data on the coupled process $\pi \pi \rightarrow K \bar{K}$ are not well described, above $1.15 \mathrm{GeV}$ even qualitatively. The combined analysis of the considered coupled processes is needed, which is also carried out satisfactorily. Then both above-indicated deficiencies related to the one-channel analysis are removed. The most important change concerns parameters of the $f_{0}(600)$ which are now in some accordance with the prediction by Weinberg on the basis of mended symmetry. The obtained $\pi \pi$ scattering length agrees well with the values extracted from the data on $K_{e 4}$ decay and DIRAC experiment and also with results from ChPT.
\end{abstract}

\section{Introduction}

The problem of interpretation of scalar mesons is tightly related to the most profound topics in particle physics which concern the QCD vacuum. Parameters of the scalar mesons, their nature and status of some of them are still not well settled. E.g., applying our model-independent method in the 3channel analyses of processes $[1,2]$ we have obtained parameters of the $f_{0}(600)$ and $f_{0}(1500)$ which differ considerably from results of analyses utilizing other methods (mainly those based on dispersion relations and Breit-Wigner approaches). Reasons for this difference should be understood because our method of analysis is based only on the demand for analyticity and unitarity of the amplitude using a uniformization procedure. The construction of the amplitude is essentially free from any dynamical (model) assumptions utilizing only the mathematical fact that a local behaviour of analytic functions determined on the Riemann surface is governed by the nearest singularities on all corresponding sheets.

\section{The uniformizing variable method}

The 2-channel S-matrix is determined on the 4-sheeted Riemann surface. The matrix elements have the right-hand cuts along the real axis of the $s$ complex plane, starting with the channel thresholds and the left-hand cuts related to the crossed channels. Structure of the Riemann surface is taken into

\footnotetext{
a e-mail: surovcev@theor.jinr.ru
}

This is an Open Access article distributed under the terms of the Creative Commons Attribution License 2.0, which permits unrestricted use, distribution, and reproduction in any medium, provided the original work is properly cited. 
account in the uniformizing variable $v$ in which the $\pi \pi$ and $K \bar{K}$ threshold branch-points and the lefthand branch-point at $s=0$ are considered. Resonance representations are obtained from the formulas for the analytic continuation of the S-matrix elements to unphysical sheets expressed in terms of the matrix elements on the physical (I) sheet having only resonance zeros [2]. Arrangement of poles and zeros on the Riemann surface representing a resonance is denoted as a pole cluster. In the 2-channel case, there are only three types of clusters, e.g., the $f_{0}(600)$ is represented by the cluster of type (a) generated with a zero only in the matrix element $S_{11}$ on the physical sheet [2].

The S-matrix elements $S_{i j}(i, j=1,2)$ are parameterized via the Le Couteur-Newton relations. The main model-independent contribution of resonances, given by the pole clusters, is factorized in the matrix elements from the background. Possible remaining small (model-dependent) contributions of resonances are included in the background. The background is parameterized by means of complex energy-dependent phases which model the influence of the $\eta \eta, \sigma \sigma$, and an effective ( $\eta \eta^{\prime}$, $\rho \rho, \omega \omega)$ channels. The threshold of the $\sigma \sigma$ and effective channels were determined in the analysis: $s_{\sigma}=1.656 \mathrm{GeV}^{2}, s_{v}=2.129 \mathrm{GeV}^{2}$. On the uniformization plane, $S_{11}(v)$ has no cuts but $S_{12}(v)$ and $S_{22}(v)$ do have the cuts which arise from the left-hand cut on the $s$-plane, starting at $s=4\left(m_{K}^{2}-m_{\pi}^{2}\right)$. This structure is approximated with a 4 th-power pole $[3,4]$.

The resonance zeros and background parameters are fixed by fitting the phase shifts and inelasticity parameters to data on the processes $\pi \pi \rightarrow \pi \pi, K \bar{K}$ [4]. Two alternative data sets, set I and II, were considered varying in selection of the $\pi \pi$ scattering data in the region $0.58 \mathrm{GeV}<\sqrt{s}<1.89 \mathrm{GeV}$ [4].

\section{Results and discussion}

In the analysis of only $\pi \pi$ scattering we obtained an excellent description of the data set I for the phase shift and inelasticity with $\chi^{2} / n . d . f .=1.08$ [4]. The resonance parameters largely agree with the values cited in the PDG tables, e.g., the pole for the $f_{0}(600)$ on sheet II coincides well with that around $450-$ $i 275 \mathrm{MeV}$ found in the dispersive $\pi \pi$-scattering data analyses [5,6]. More remarkable differences are found for the mass of $f_{0}(980), 1001 \mathrm{MeV}$ compared to $980 \pm 10 \mathrm{MeV}$ of PDG, and for the width of $f_{0}(1500), 336 \mathrm{MeV}$ as against $109 \pm 7 \mathrm{MeV}$ of PDG. The mass of $f_{0}(980)$ slightly above $1 \mathrm{GeV}$ was also obtained in other works which analyzed the $\pi \pi$ scattering [7]. We interpret the broad $f_{0}(1500)$ as a superposition of two states, broad and narrow. The narrow state is observed in processes considered in works cited by the PDG. To check our interpretation we re-fitted the $\pi \pi$-scattering data including both broad and narrow $f_{0}(1500)$. We obtained an improved description, $\chi^{2} / n . d . f .=1.07$, in which the parameters of the narrow $f_{0}(1500)$ are consistent with those in the PDG tables. The parameters of the other resonances practically did not change. The $\pi \pi$ scattering length $a_{0}$, obtained in these one-channel analyses is $a_{0}=(0.222 \pm 0.008) m_{\pi}^{-1}$, which is also in a very good agreement with the experimental values and with the results of the ChPT calculations, see Ref. [4] and references therein.

However, let us emphasize two important flaws of this description: 1. The negative phase-shift in the background beginning from the $\pi \pi$ threshold $\left(a_{11}=-0.0895\right)$ is necessary for a successful description of the data. This should not be the case because, in the uniformizing variable, we have included the left-hand branch-point at $s=0$ which gives the main contribution to the $\pi \pi$ background below the $K \bar{K}$ threshold. Other possible contributions of the left-hand cut related with exchanges of the nearest mesons, the $\rho$ and $f_{0}(600)$, practically obliterate each other because the vector and scalar particles contribute with the opposite signs due to the gauge invariance. 2. Description of the data on reaction $\pi \pi \rightarrow K \bar{K}$, using the same parameters of resonances as in the $\pi \pi$ channel, is satisfactory only for the phase shift [4] which is due to the approximation of the left-hand branch-point at $s=4\left(m_{K}^{2}-m_{\pi}^{2}\right)$ in $S_{12}$ and $S_{22}$ by the 4th-power pole. The $\left|S_{12}\right|$ is described well only from the $K \bar{K}$ threshold to about $1.15 \mathrm{GeV}$ as it should be due to the two-channel unitarity. Above this energy the description fails even qualitatively [4]. Therefore, if the data are consistent, for obtaining correct parameters of broad multi-channel resonances a combined analysis of data on the coupled processes $\pi \pi \rightarrow \pi \pi, K \bar{K}$ is needed.

In the combined analysis of data set I, the resonances are described by pole clusters of the same types as in the analysis only of $\pi \pi$-scattering [4] with a satisfactory result, the total $\chi^{2} / n . d . f .=1.44$. The data for the $\pi \pi$ scattering below $1 \mathrm{GeV}$ admit two solutions for the phase shift: A and B which 
differ mainly in the pole position on sheet II for the $f_{0}(600)$. The $\chi^{2} / n$.d.f. shown above is for the solution $\mathrm{B}$. The A-solution gives a slightly worse value 1.53. Positions of poles of the resonance clusters on the $s$-plane for the solutions A and B are similar within the magnitudes of errors except for the $f_{0}(600)$ [4], e.g., on the sheet II the positions for $f_{0}(600)$ are $517.0 \pm 7.8-i(393.9 \pm 6.0) \mathrm{MeV}$ and $550.6 \pm 9.0-i(502.1 \pm 7.2) \mathrm{MeV}$ for the solutions $\mathrm{A}$ and $\mathrm{B}$, respectively. In both solutions the phase shift in the background is zero below the inelastic threshold, $a_{11}=0 \pm 0.003$, and the $\pi \pi \rightarrow K \bar{K}$ data are described satisfactorily unlike the case using the resonance parameters from the only $\pi \pi$ channel analysis discussed above [4]. Therefore, in the combined analysis of data both above-indicated flaws, related to the only $\pi \pi$-scattering analysis, are amended. Now the $\pi \pi$ background below the $K \bar{K}$ threshold is absent because its contribution is practically completely allowed for by the left-hand branch-point at $s=0$ included in the uniformizing variable. An arising pseudo-background at the $\eta \eta$ threshold $\left(a_{1 \eta}<0\right)$ is also clear: this is a direct indication that the $\eta \eta$-threshold branch-point should be considered explicitly in the uniformizing variable. This was recently done in the 3-channel analysis [2].

In the combined analysis of data set II, the resonances are described by the pole clusters of the same types as in the previous case except for the narrow $f_{0}(1500)$ which is represented now by the cluster of type (a) [2] to provide a more rapid growth of the $\pi \pi$ phase shift above $1.45 \mathrm{GeV}$ than in set I. Description of the phase shifts and inelasticity parameters in both channels is also satisfactory [4], with the total $\chi^{2} / n . d . f .=1.58$ for the solution A and $\chi^{2} / n . d . f .=1.42$ for the solution B.

The $\pi \pi$ scattering length $a_{0}$ and slope parameter $b_{0}$ are calculated using the expansion of the phase shift around the $\pi \pi$ threshold [4]. The scattering lengths for the B-solutions, $a_{0}=0.282 \mathrm{~m}_{\pi}^{-1}$ (set I) and $0.275 m_{\pi}^{-1}$ (set II), agree well with the predictions of the chiral theory with linear realization of chiral symmetry (models of NJL type), whereas those for the A-solutions, $a_{0}=0.230 m_{\pi}^{-1}$ (set I) and $0.226 m_{\pi}^{-1}$ (set II) accord with predictions of ChPT (non-linear realization). Considering very precise values obtained from the data on the $K_{e 4}$ decay for $a_{0}$ and $a_{2}$ [8] and from the DIRAC experiment at CERN [9] which are rather consistent with the smaller values of $a_{0}$, one ought to prefer the solution A.

The extracted slope parameters $b_{0}$ vary from $0.201 \mathrm{~m}_{\pi}^{-3}$ to $0.210 \mathrm{~m}_{\pi}^{-3}$ for the solutions A and B with the data sets I and II [4]. These values are smaller then, e.g. the result from the analysis based on Roy-like equations: $b_{0}=0.278 m_{\pi}^{-3}[6]$.

In conclusion, let us point out that a structure of the Riemann surface of the S-matrix for considered coupled processes must be included properly. For calculating resonance parameters, such as the mass, total width and coupling with channels, one must use poles on those sheets where they are not shifted due to the channel coupling since they correctly respects the zero positions on the physical sheet. In the 2-channel case, the relevant poles are on sheets II or/and IV in dependence on the resonance type. Moreover, the combined analysis of coupled processes is needed as the analysis of only $\pi \pi$ channel do not give correct values of resonance parameters even if the Riemann surface structure is included.

The work was supported by the Heisenberg-Landau Program, the RFBR Grant 10-02-00368-a, the VotrubaBlokhintsev Program for Cooperation of Czech Republic with JINR, the Grant Agency of Czech Republic, Grant P203/12/2126, the Grant Program of Plenipotentiary of Slovak Republic at JINR, the Bogoliubov-Infeld Program for Cooperation of Poland with JINR, and partly also by the Polish Ministry of Science and Higher Education, Grant No N N202 101368.

\section{References}

1. Yu.S. Surovtsev, P. Bydžovský, R. Kamiński, M. Nagy, Phys. Rev. D 81, (2010) 016001

2. Yu.S. Surovtsev, P. Bydžovský, V.E. Lyubovitskij, Phys. Rev. D 85, (2012) 036002

3. Yu.S. Surovtsev, D. Krupa, M. Nagy, Eur. Phys. J. A 15, (2002) 409

4. Yu.S.Surovtsev, P.Bydžovský, R.Kamiński, V.E.Lyubovitskij, M.Nagy, ArXiv:1206.3438 [hep-ph]

5. G. Colangelo, J. Gasser, H. Leutwyler, Nucl. Phys. B 603, (2001) 125; B. Ananthanarayan, G. Colangelo, J. Gasser, H. Leutwyler, Phys. Rept. 353, (2001) 207

6. R.García-Martín et al., Phys. Rev. D 83, (2011) 074004

7. R. García-Martín, R. Kamiński, J.R. Peláez, J. Ruiz de Elvira, Phys. Rev. Lett. 107, (2011) 072001

8. J.R. Batley et al., Eur. Phys. J. C 70, (2010) 635

9. B. Adeva et al., Phys. Lett. B 674, (2009) 11 